\title{
Protostar Formation in the Early Universe
}

\section{Citation}

Yoshida, Naoki. 2008. "Protostar Formation in the Early Universe." Proceedings of the International Astronomical Union 4 (S255): 18-23. https://doi.org/10.1017/s1743921308024538.

\section{Permanent link}

http://nrs.harvard.edu/urn-3:HUL.InstRepos:41381772

\section{Terms of Use}

This article was downloaded from Harvard University's DASH repository, and is made available under the terms and conditions applicable to Open Access Policy Articles, as set forth at http:// nrs.harvard.edu/urn-3:HUL.InstRepos:dash.current.terms-of-use\#OAP

\section{Share Your Story}

The Harvard community has made this article openly available.

Please share how this access benefits you. Submit a story.

Accessibility 


\title{
Protostar Formation in the Early Universe
}

\author{
Naoki Yoshida, ${ }^{1}$ Kazuyuki Omukai, ${ }^{2}$ Lars Hernquist ${ }^{3}$ \\ ${ }^{1}$ Department of Physics, Nagoya University, Furocho, Chikusa, Nagoya, Aichi 464-8602, Japan \\ ${ }^{2}$ National Astronomical Observatory of Japan, Osawa, Mitaka, Tokyo 181-8588, Japan \\ ${ }^{3}$ Harvard-Smithsonian Center for Astrophysics, \\ 60 Garden Street, Cambridge, MA02138 \\ *To whom correspondence should be addressed; E-mail: nyoshida@a.phys.nagoya-u.ac.jp. \\ Science, 321, 669 (2008)
}

The nature of the first generation of stars in the Universe remains largely unknown. Observations imply the existence of massive primordial stars early in the history of the universe, and the standard theory for the growth of cosmic structure predicts that structures grow hierarchically through gravitational instability. We have developed an $a b$ initio computer simulation of the formation of primordial stars that follows the relevant atomic and molecular processes in a primordial gas in an expanding universe. The results show that primeval density fluctuations left over from the Big Bang can drive the formation of a tiny protostar with a mass of just one percent that of the Sun. The protostar is a seed for the subsequent formation of a massive primordial star.

Large ground-based telescopes have discovered distant astronomical objects such as galaxies and quasars (1, 2) that were in place when the Universe was less than 1 billion 
years old, or about $5 \%$ of its current age. Moreover, these studies have shown that other luminous objects must have been present even earlier. For example, the most distant known quasar, SDSS-J1148, contains substantial amounts of heavy elements such as carbon, oxygen, and iron as well as dust grains (3). These heavy elements are not of cosmic origin, but must have been formed earlier in massive stars before being expelled by supernovae and stellar winds, and then incorporated into the material that later condensed to produce this quasar.

Recently, stars with extremely low heavy element content were discovered in the halo of our Galaxy (4, 5). The observed elemental abundance patterns indicate several possibilities for the nature of their ancestors (6, 7). One interesting scenario is that supernova explosions of massive primordial stars enriched the parent gas clouds, from which these halo stars were born.

Theoretical analyses hold promise for revealing the process of primordial star formation for two main reasons: (i) the initial conditions, as determined cosmologically, are well-established, so that statistically equivalent realizations of a standard model universe can be accurately generated, and (ii) the important basic physics such as gravitation, hydrodynamics, and atomic and molecular processes in a hydrogen-helium gas are understood. Other complications that plague investigations of star formation in the local Universe, such as the presence of strong magnetic fields or heavy elements, can be neglected at these early times.

Here, we report super-computer simulations of the development of cosmic structure in the early Universe and the formation of primordial stars. Our simulations achieve a dynamic range in spatial scale of $\sim 10^{13}$, resolving small-scale structures having sizes of a fraction of a solar radius $\left(\sim 10^{10} \mathrm{~cm}\right)$ within cosmological volumes hundreds of kiloparsecs in length $\left(\sim 10^{23} \mathrm{~cm}\right)$. The smallest length scale, the so-called local Jeans length set by the 
action of gravity and hydrodynamic pressure, is fully resolved throughout the simulation volume at all times.

We do not assume any a priori equation of state for the gas. The thermal and chemical evolution of the gas is determined fully by molecular and atomic processes, which are treated in a direct, self-consistent manner. The spatial resolution and the accurate implementations of the physical processes allow us to follow the collapse of a gas to stellar densities, and thus our calculations offer a detailed picture of how the first cosmological objects - primordial protostars - form from chemically pristine gas.

We set up cosmological initial conditions such that the statistical properties of the density and velocity fields are matched to those given by the standard model of the Universe (8), according to which the energy density is dominated by dark energy and cold dark matter. We follow the gravitational collapse of dark matter and the hydrodynamics of primordial gas through simulations of cosmic structure formation. Below, we provide details on one simulation, which followed the evolution of dark matter and gas in a cube 200 comoving kiloparsecs on a side. We focus our attention on a gravitationally bound dark matter halo which formed in this volume at an epoch when the cosmological redshift was $z=14$ (Fig. 1). The mass of this halo, a half million solar masses, and the physical conditions within it are particularly conducive for it to host a primordial star (9, 10, 11). The gas within this halo had a temperature of $\sim 1000$ Kelvin and a small mass of hydrogen molecules ( $\sim 10^{-4}$ in number fraction) had already formed, enabling efficient radiative cooling.

Through the action of radiative cooling, a star-forming gas cloud collected in the host dark halo. We tracked the subsequent thermal and chemical evolution of the primordial gas cloud for more than 20 decades in density up to the epoch of protostar formation. We accounted for: (i) the chemistry at both low and high densities, including molecular 
hydrogen formation, (ii) transfer of molecular line photons and accompanying radiative cooling, (iii) transfer of collisionally-induced continuum radiation and resulting radiative cooling. In the final phase of the collapse, the temperature increased adiabatically as a result of the absence of radiative and chemical cooling. This continued until the contraction of the central part was halted by strong thermal pressure. At this time, strong hydrodynamic shocks formed, marking the moment of the formation of a protostar. We could not follow the evolution after this epoch accurately without implementing radiative effects from the post-shock high temperature gases, and thus we stopped the simulation at this point.

The structure in and around the newly formed protostar wass rather complex (Fig. 1 D). At this time, there were substantial variations in density and temperature even in the innermost 10 solar-radii region. Clearly, the primordial protostar was not simply a sphere surrounded by a single accretion shock.

The cloud evolution was dictated by several important physical processes: First, a fully molecular cloud with mass $\sim 1 M_{\odot}$ formed $(10,12,13)$ when the gas density was sufficiently high $\left(>10^{8} \mathrm{~cm}^{-3}\right)$ that three-body chemical reactions converted nearly all the hydrogen into molecules. Efficient cooling by rovibrational transitions of hydrogen molecules caused the first small dip at a radius of $R \sim 10^{16} \mathrm{~cm}$ in the radial temperature profile (Fig. 2). When the dense, molecular part contracted further, it became optically thick to hydrogen molecular rovibrational lines, and then the efficiency of radiative cooling saturated (dotdashed lines in Fig. 2). At still higher densities, frequent collisions between hydrogen molecules led to efficient emission of continuum radiation via collision-induced emission. By this rapid cooling occurring at densities greater than $\sim 10^{14} \mathrm{~cm}^{-3}$, a small central part with $\sim 0.1 M_{\odot}$ cooled efficiently to form a flattened disk-like structure. In the flattened gas cloud, radiation escaped preferentially in the direction where contraction was fastest, 
because the velocity gradient was large and also the continuum optical depth was small in this direction [supporting online material (SOM) text and Fig. S1, S2]. This combined effect of gravitational contraction and direction-dependent radiative cooling accelerated the deformation of the cloud core to a disk structure. The disk structure had a radius of $\sim 10^{13} \mathrm{~cm}$ and a mass of $\sim 0.1 M_{\odot}$, where the cooling time and the local dynamical time are comparable. While the innermost portion further contracted slowly, spiral density waves were excited, yielding two arms (see the bottom-right panel of Fig. 1).

When the central density reached $n \sim 10^{18} \mathrm{~cm}^{-3}$, the gas became completely optically thick to continuum radiation, and at this point radiative cooling no longer operated efficiently (long dashed lines in Fig. 2). Further collapse and the associated dynamical heating triggered full-scale dissociation of hydrogen molecules in the central part (see also Fig. 3). After most of the hydrogen molecules were collisionally dissociated, the gas could not lose its thermal energy either radiatively or by dissociating molecules. The resulting effective equation of state became progressively more stiff, making the gas cloud resist gravitational deformation and fragmentation (14, 15). The gas then contracted adiabatically, and its temperature quickly increased above several thousand Kelvin, while the density exceeded $n \sim 10^{20} \mathrm{~cm}^{-3}$. The strong thermal pressure finally stopped the gravitational collapse and hydrodynamic shocks were generated (solid lines in Fig. 2). We define a constant density, atomic gas core as a protostar that is pressure-supported. At the final output time, a protostar formed with a mass of just 0.01 solar masses. It had an initial radius of $\sim 5 \times 10^{11} \mathrm{~cm}$, similar to that of present-day protostars in theoretical calculations (16). The central particle number density of the protostar was $\sim 10^{21} \mathrm{~cm}^{-3}$ and the temperature was well above 10,000 K.

At the time of protostar formation, the central temperature was so high that almost all the molecules were collisionally dissociated within an enclosed mass of $0.01 M_{\odot}$ (Fig. 3). 
A slight degree of ionization was also seen in the innermost high-pressure part of the atomic core. There was a small variation in the atomic hydrogen fraction at $\sim 0.1 M_{\odot}$; a small fraction of hydrogen molecules were dissociated when the gas temperature reached $\sim 2000 \mathrm{~K}(12,17)$, but the molecular fraction increased again when the density increased and efficient continuum cooling brought the gas temperature temporarily below $2000 \mathrm{~K}$ (see the second temperature dip at $R \sim 10^{13} \mathrm{~cm}$ in Fig. 2).

The protostar accretes the ambient gas in a complicated way (Fig. 3 bottom). In the direction vertical to the disk plane, gas falling in at a velocity exceeding $10 \mathrm{~km} / \mathrm{sec}$ was suddenly stopped at the location of a shock at $M_{\text {enclosed }} \sim 0.01 M_{\odot}$. Fig. 3 also shows the degree of rotational support of the gas, defined as $f_{\text {rot }}=(L / r) / v_{\text {Kep }}$, where $L$ is the specific angular momentum of the gas within radius $r$, and $v_{\text {Kep }}$ is the Keplerian velocity at that radius.

Within a mass of $\sim 0.1 M_{\odot}$, two spiral arms rotated rapidly, and the outer part $\left(\sim 0.05-0.1 M_{\odot}\right)$ appeared nearly centrifugally supported, whereas the central part had gravitationally collapsed. The central core lost part of its angular momentum via gravitational torques exerted by non-axisymmetric perturbations. The newly-born protostar was supported by both thermal pressure and rotation. The overall velocity structure is characteristic of a collapsing gas with an initially slow rotation, as reported in previous studies of both present-day and primordial star-formation (18, 19).

A long-standing question is whether a primordial gas cloud such as that studied here experiences vigorous fragmentation by thermal instability during its evolution. In our simulation, a single small proto-stellar core formed first and the central part did not fragment into multiple objects before protostar formation. At all phases, the locally estimated growth time for isobaric perturbations was longer than, or only comparable to, the local dynamical time for collapse. Hence, the cloud core did not fragment by thermal 
instability, but instead its collapse accelerated.

It has been suggested that the central part of primordial gas clouds may break up into smaller clumps later during the subsequent accretion phase (20, 19). We have examined a core fragmentation model of $(\underline{18})$ by measuring $\Omega t_{\text {dyn }}$ where $\Omega$ is the mean angular velocity and $t_{\mathrm{dyn}}=1 / \sqrt{4 \pi G \rho}$ is the local dynamical time. The central $\sim 0.01 M_{\odot}$ portion had a value of $\Omega t_{\mathrm{dyn}}=0.25$, which is large and close to the critical value for fragmentation. Thus the formation of multiple stellar systems may be possible, although not very likely, during later accretion phases.

The instantaneous gas mass accretion rate at the time of protostar formation was as large as $0.01-0.1$ solar masses per year within the innermost $10 M_{\odot}$. If the gas in the inner portion accreted efficiently, the protostar would quickly grow to be as massive as 10 solar masses within a thousand years (21). Even if multiple stellar seeds formed, there would be at least one main accreting protostar. A detailed proto-stellar calculation for a similarly large accretion rate predicts that the mass of the star when it lands on the main sequence will be $\sim 100 M_{\odot}(22,13)$.

Feedback effects, in particular those from ionizing photons emitted by the protostar, work to evaporate the surrounding gas and to halt gas accretion. A semi-analytic calculation including this radiative feedback and the effect of rotation shows that the final stellar mass can still be greater than a few tens of solar masses in a reasonable parameter space of the model (23). If instead mass accretion is unimpeded throughout the star's evolution, the final stellar mass can be very large, possibly exceeding a few hundred solar masses (22, 21). Such very massive stars ionize a large volume of the surrounding gas. Because of the different thermal evolution of an initially ionized gas (24), second generation primordial stars formed under such conditions are predicted to be several tens of solar masses (25, 26). Therefore, in either case, our model provides a viable scenario for 
the early chemical enrichment in the Universe by massive primordial stars (6), which is necessary for the formation of later populations of ordinary stars.

The basic properties of the particular star-forming cloud we simulate, such as physical size and mass, are characteristic for cosmological primordial gas clouds, and the object is indeed similar in many aspects to those found in previous works $(10,11,27)$. The final evolution of the central high-density part will likely be affected by its angular momentum content (19). However, because the bulk of the cloud core is assembled from material with low angular momentum (13), it generally has a slow initial spin, and thus the evolution of prestellar gas is expected to be similar to what is presented here. Our simulation thus offers a complete picture of how a primordial protostar may have formed from tiny cosmological density fluctuations. Primordial star formation for different cosmological models has been explored (28, 29). The particle properties of dark matter may be another important factor in star-formation in the early universe.

\section{References and Notes}

1. X. Fan et al., Astronomical J. 125, 1649 (2003).

2. M. Iye et al., Nature 443, 186 (2006).

3. F. Walter et al., Nature 424, 406 (2003).

4. N. Christlieb et al., Nature 419, 904 (2002).

5. A. Frebel et al., Nature 434, 871 (2005).

6. N. Iwamoto et al., Science 309, 451 (2005).

7. Y. Komiya et al., Astrophys. J. 658, 367 (2007). 
8. D. N. Spergel et al., Astrophys. J. 424, 406 (2007).

9. V. Bromm, P. S. Coppi, R. B. Larson, Astrophys. J. 527, 5 (1999)

10. T. Abel, G. L. Bryan, M. N. Norman, Science 295, 93 (2002)

11. V. Bromm, P. S. Coppi, R. B. Larson, Astrophys. J. 564, 23 (2002)

12. K. Omukai, R., Nishi, Astrophys. J. 508, 141 (1998).

13. N. Yoshida, K. Omukai, L. Hernquist, T. Abel, Astrophys. J. 652, 6 (2006).

14. T. Hanawa, T. Matsumoto, Pub. Astron. Soc. Japan 52, 241 (2000).

15. K. Omukai, T. Tsuribe, R. Schneider, A. Ferrara, Astrophys. J. 626, 627 (2005).

16. R. B. Larson, Mon. Not. R. Astron. Soc. 145, 271 (1969).

17. E. Ripamonti, F. Haardt, A. Ferrara, M. Colpi, Mon. Not. R. Astron. Soc. 334, 401 (2002).

18. T. Matsumoto \& T. Hanawa, Astrophys. J. 595, 913 (2003).

19. M. N. Machida, K. Omukai, T. Matsumoto, S. Inutsuka, Astrophys. J. 677, 813 (2008).

20. P. C. Clark, S. C. O. Glover, R. S. Klessen, Astrophys. J. 672, 757 (2008).

21. V. Bromm, A. Loeb, New Astronomy 353, 364 (2004).

22. K. Omukai \& F. Palla, Astrophys. J. 589, 677 (2003).

23. C. F. McKee, J. Tan, Astrophys. J. 681, 771 (2008)

24. H. Uehara, S. Inutsuka, Astrophys. J. Lett. 531, 91 (2000). 
25. J. L. Johnson, V. Bromm, Mon. Not. R. Astron. Soc. 366, 247 (2006).

26. N. Yoshida, K. Omukai, L. Hernquist, Astrophys. J. 667, 117 (2007).

27. B. O'Shea, M. L. Norman, Astrophys. J. 654, 66 (2007).

28. L. Gao, T. Theuns, Science 317, 1527 (2007).

29. D. Spolyar, K. Freese, P. Gondolo, Phys. Rev. Lett. 100, 1101 (2008).

30. We thank M. Machida and T. Matsumoto for fruitful discussions on gas cloud evolution. N.Y., K.O. have been supported in part by the Grants-in-Aid for Young Scientists 17684008, 18740117, 19047004 by the Ministry of Education, Culture, Science and Technology of Japan, and by the Mitsubishi Foundation. 


\section{(A) cosmological halo}

\section{0 parsec}

\section{(D) new-born protostar}

\section{5 solar-radii}

Fig. 1: Projected gas distribution around the protostar. Shown regions are, from top-left, clockwise, (A) the large-scale gas distribution around the cosmological halo (300 pc on a side), (B) a self-gravitating, star-forming cloud (5 pc on a side), (C) the central part of the fully molecular core (10 astronomical units on a side), and (D) the final protostar (25 solar-radii on a side). We use the density-weighted temperature to color (D), to show the complex structure of the protostar. 

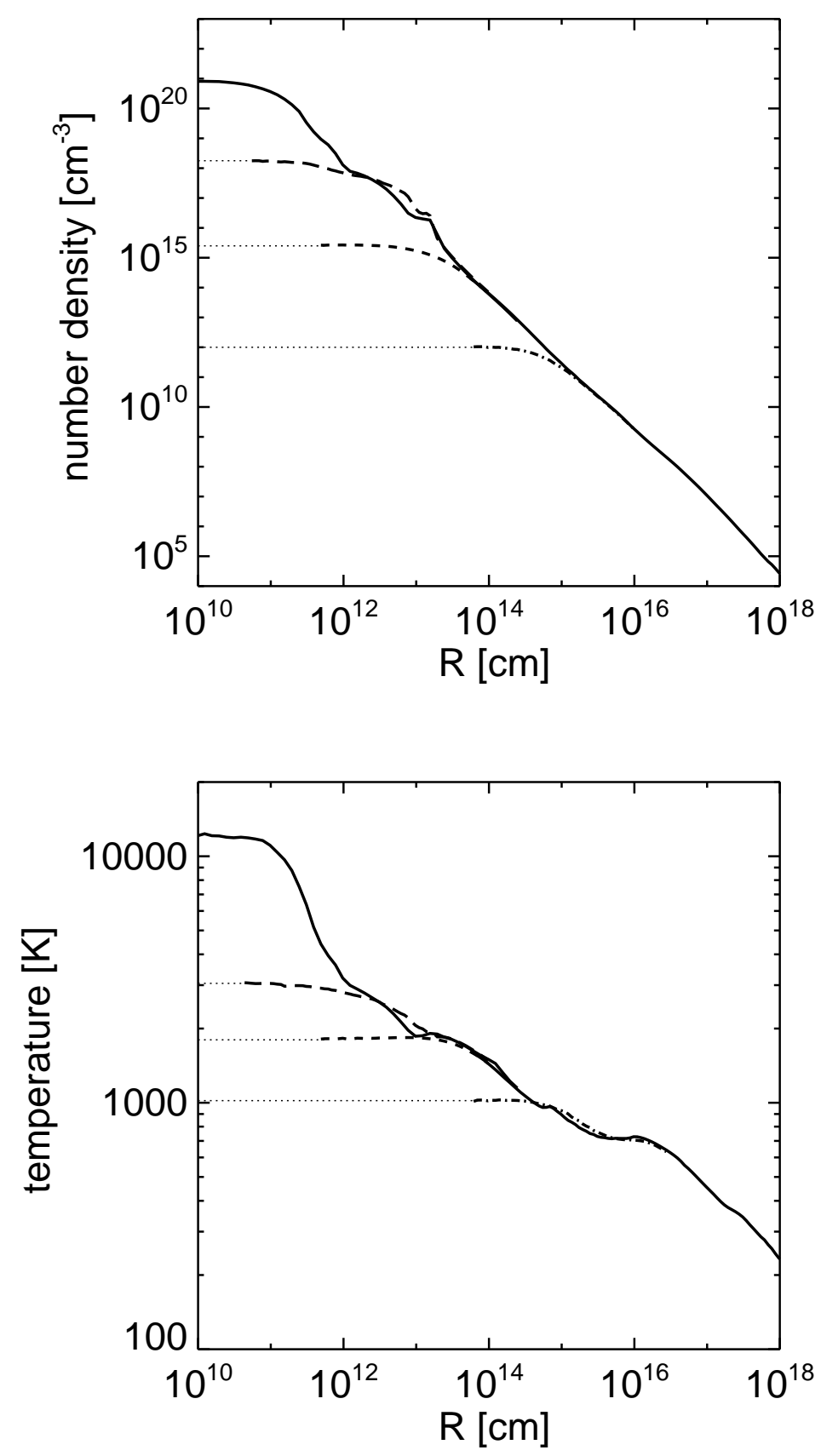

Fig. 2: Evolution of spherically-averaged radial density profile (top) and temperature profile (bottom) around the protostar. Epochs are shown when the central core became optically thick to molecular lines (dot-dashed lines), when cooling by collision-induced emission kicked-in (short dashed lines), when the core became optically thick to continuum (long dashed lines), and when full-scale dissociation was completed and a pressuresupported core formed (solid lines). 

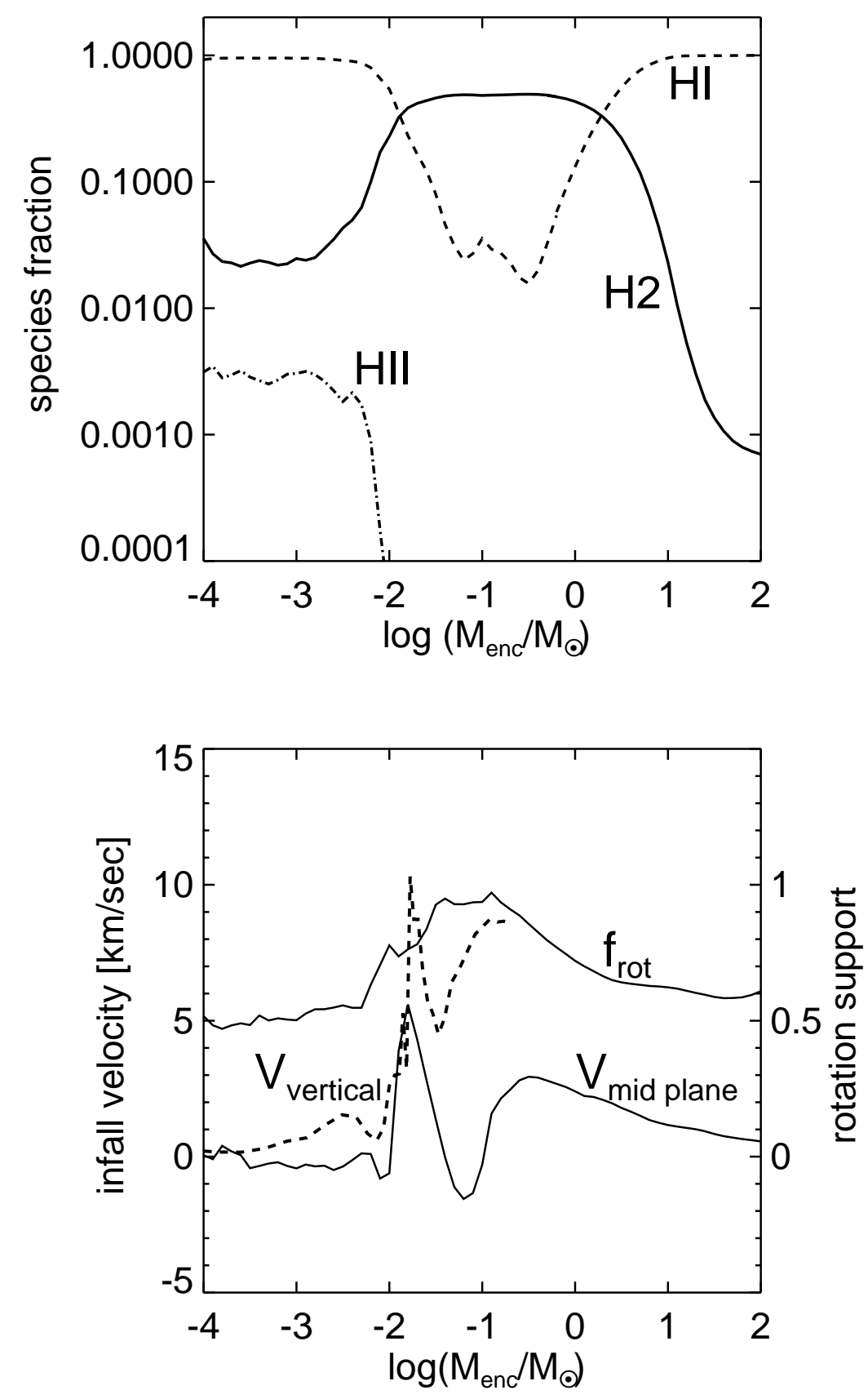

Fig. 3: Structure of the protostar. (Top panel) The number fractions of atomic hydrogen (dashed line), molecular hydrogen (solid line), and ionized hydrogen (dot-dashed line). (Bottom panel) The gas infall velocity in one direction perpendicular to the disk plane (dashed line) and an azimuthally averaged inflow velocity at the midplane (solid line). The thin solid line shows the degree of rotational support as defined in the text. 